\title{
Effect of Locations of using High Boost Filtering on the Watermark Recovery in Spatial Domain Watermarking
}

\author{
Mehdi Alirezanejad ${ }^{1 *}$, Vahid Saffari², Saeed Amirgholipour ${ }^{3}$, Aboosaleh Mohammad Sharifi ${ }^{3}$ \\ 'Department of Computer,Firuzkooh Branch, Islamic Azad University,Firuzkooh, Iran; \\ m_arn2004@yahoo.com \\ 2Department of Computer, Collage of Engineering, Shiraz branch, Islamic Azad University, Shiraz, Iran; \\ Saffari@iaushiraz.ac.ir \\ ${ }^{3}$ Department of Computer, Ramsar Branch, Islamic Azad University, Ramsar, Iran; \\ s.kasmani@gmail.com, sharifi@iauramsar.ac.ir
}

\begin{abstract}
Generally, High Boost filter is used to emphasize high frequency components representing the image details without eliminating low frequency components representing the basic form of the signal. The dissimilarity between the watermark and unwatermarked parts of the image are increased by this filter. Thus, watermark could be recovered significantly better by recovery algorithm. In this paper, a comparison is taken place between the effects of different places of preforming High Boost filter on the reliability of the recovery methods in spatial domain image watermarking. This filter is convolved in two different places in the watermark extraction algorithm; before running the watermark extraction and before calculate the correlation in the middle of extraction. This paper concentrate to conclude which of these places is more proper to convolve this filter. A classic correlation based method is used as a representative of spatial domain watermarking. Several experiment are done to show which one of this places is more proper for preforming proposed filter.
\end{abstract}

Keywords: Correlation Based Watermarking, Digital Image Watermarking, High Boost Filter, Spatial Domain Watermarking.

\section{Introduction}

The watermarking has been proposed as an appropriate solution for copyright protection methods for digital data. Different watermarking methods embed a watermark with some extra information about the digital media without visibly modifying them ${ }^{1}$. Digital watermarking methods are consisting of two parts; embedding and extracting algorithms. In the embedding algorithm, the watermark is embedded in digital media with a special method. And the embedded watermark is recovered from the watermarked image in extracting algorithm.

Resistant and imperceptibility are two important factors in watermarking algorithm, and these properties are incompatible with each other. The imperceptibility means that how much the media quality is changed by the embedded watermark; and the robustness is the ability to recover the watermark after common or malicious signal processing operations. These incompatible parameters should be set to meet the requirements of the application ${ }^{2,3}$.

Generally watermark is embedded in the spatial domain or in the transform domain. The watermark is embedded straight forward in the pixels of the host image in the spatial domain methods. But, these methods are not robust to image common image processing operation $^{4}$. Although the filters capability are used to increase the power of recovery algorithm ${ }^{3,5,6}$, Transform domain watermarking methods take advantage of the transform domain properties to embed the watermark. These methods usually use the Discrete Cosine Transform $(\mathrm{DCT})^{7,8}$ and the Discrete Wavelet Transform (DWT) $)^{9,10}$. These methods typically are more imperceptible and robustness to common image processing operation.

*Author for correspondence 
Many researchers have tried to increase the power of watermarking algorithms. Several of these researchers concentrate on finding most suitable places for watermark embedding, and the others have studied the enhancement algorithm to increase power of watermark retrieval algorithms.

In the first method, there are attempts to use human visual system characteristics to choose appropriate for resistance and transparency. These techniques generally utilize the frequency-domain characteristics to detect the most proper locations to embed the watermark. These methods are commonly applied on frequency domain watermarking techniques. There are several methods which are based on probing the most suitable coefficients to embed watermark information ${ }^{4}$. These approaches utilize a perceptually optimal quantization matrix ${ }^{11}$, Just Noticeable Difference (JND) $)^{12,13}$, wavelet filter ${ }^{14}$, and Human Visual System (HVS) ${ }^{15-17}$, for probing the most suitable coefficients to embed the watermark information.

However, in the second methods, researchers try to make watermark information more visible to the recovery algorithms. Therefore, these approaches could increase the capability of recovery algorithms. The goal of these methods is to boost watermark detection rate by an efficient and simple model based on image enhancement, edge detection and noise magnifying methods ${ }^{3,4}$.

An improved detector is proposed for detection based on thresholds extracted by statistic rules on which the method relies ${ }^{18}$. Application of blurring filter for increasing the possibility of detection of watermark is proposed by Braudaway ${ }^{19}$. They use blurring filters for a watermark with a dominant low frequency content to improve the detection probability. Since the content of image might interfere with the watermark, especially in the lowfrequency parts, the reliability of the detector could be improved by applying matched filtering before correlation $^{6}$. This decreases the influence of the original image to the correlation. Therefore, the watermark could easily be recovered from watermarked image.

Hafiz proposed an approach to blind watermark detection for spread spectrum by using of Independent Component Analysis theor ${ }^{20}$. It uses the theory of Independent Component Analysis (ICA) and detects the watermark with a blind source separation method. The watermark information is considered as noise for the watermarked image in its spatial domain. This noise is magnified before executing recovery algorithm, and then recovers the watermark information by adjusting the extracted data from the frequency domain according to the global minimum method ${ }^{21}$. A pre-processing method is proposed that exploit a combination of noise boosting and filtering to facilitate recovering the watermark from watermarked image in the DCT-based watermarking algorithm ${ }^{3,5}$.

In this paper, a comparison is made between effects of different locations of utilizing High Boost filter on increasing power of recovery algorithms in the spatial domain methods. The dissimilarity between the watermark and the original image are increased by this filter. Thus, the watermark could be removed from the modified watermarked image meaningfully better. This filter is applied before running watermark extraction procedures, and before taking the correlation between the extracted block and pseudo random noise, in correlation based method. Several experiments are done to show that which of these locations is proper for convolving High Boost filter in the spatial domain based watermarking.

The rest of the paper is organized as follows. In section 2, High Boost filter is described in details. A typical correlation based method is introduced as a representative of spatial domain watermarking in section 3. Section 4 presents the experimental results to demonstrate the performance of this scheme on the classical method. Conclusions are drawn in Section 5.

\section{High Boost Filter}

In this paper, Gonzales definition is used for High Boost filter ${ }^{21}$.

High boost filtering can be implemented with one pass using either of the two masks shown in equation (1), (2). Note that, when $A=0$, high-boost filtering becomes "standard" Laplacian sharpening. As the value of $A$ increases past 1 , the contribution of the sharpening process becomes less and less important. Eventually, if A is large enough, the high-boost image will be approximately equal to the original image multiplied by a constant.

$$
\begin{aligned}
& {\left[\begin{array}{ccc}
0 & -1 & 0 \\
-1 & A+4 & -1 \\
0 & -1 & 0
\end{array}\right]} \\
& {\left[\begin{array}{ccc}
-1 & -1 & -1 \\
-1 & A+8 & -1 \\
-1 & -1 & -1
\end{array}\right]}
\end{aligned}
$$

One of the principal applications of High Boost filtering is when the input image is darker than desired. 
By varying the boost coefficient, it generally is possible to obtain an overall increase in average gray level of the image, thus helping to brighten the result.

In this paper, a standard type of High Boost filter is used. It is shown in the $3 \times 3$ convolution matrix looks as follows:

$$
\left[\begin{array}{ccc}
-1 & -1 & -1 \\
-1 & 9 & -1 \\
-1 & -1 & -1
\end{array}\right]
$$

\section{Correlation based} Watermarking using Block Processing in the Spatial Domain

\subsection{Watermark Embedding Algorithm}

The watermark embedding process is represented in Figure 1, followed by a detailed explanation.

Step 1: Divide the host image into $16 \times 16$ blocks.

Step 2: Re-formulate the watermark image into a vector of zeroes and ones.

Step 3: Generate two uncorrelated pseudorandom sequences by a key. One sequence is used to embed the watermark bit 0 (PN_0) and the other sequence is used to embed the watermark bit 1 (PN_1). Number of elements in each of the two pseudorandom sequences must be equal to the number of block.

Step 4: Embed the two pseudorandom sequences, PN_0 and PN_1, with a gain factor $\alpha$ in the $16 \times 16$ blocks of the host image. If we donate $\mathrm{X}$ as the matrix of the block, then embedding is done as equation (4):

$$
x=\left\{\begin{array}{l}
x+\alpha^{*} P N 0 \text { watermark_bit }=0 \\
x+\alpha^{*} \text { PN } 0 \text { watermark_bit }=1
\end{array}\right.
$$

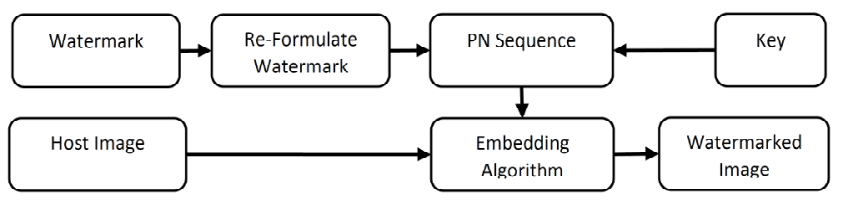

Figure 1. The watermark embedding process.

\subsection{Watermark Extracting Procedure}

The typical correlation based algorithm is a blind watermarking algorithm, and thus the original host image is not required to extract the watermark. Recovery method is the same as embedding one, and filtering is convolved before running extraction and before calculate the correlation on each block to better separate watermark information from host image. The watermark extraction procedure is shown in Figure 2, and described in details in the following steps.

Step 1:Applying High Boost filter as shown in the equation of (3) on the watermarked image.

Step 2: Divide watermarked image that could be attacked or not into $16 \times 16$ blocks.

Step 3: Regenerate the two pseudorandom sequences (PN_0 and PN_1) using the same key which used in the watermark embedding procedure.

Step 4: For each block in the watermarked image calculate the correlation between the element and the two generated pseudorandom sequences (PN_0 and PN_1). If the correlation with the PN_0 was higher than the correlation with $\mathrm{PN}_{-} 1$, then the extracted watermark bit is considered 0 , otherwise the extracted watermark is considered 1.

Step 5: The scrambled watermark is reconstructed using the extracted watermark bits.

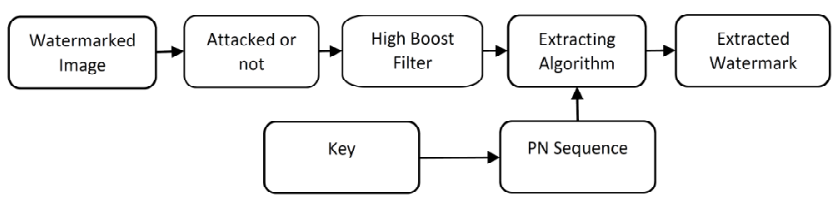

(a)

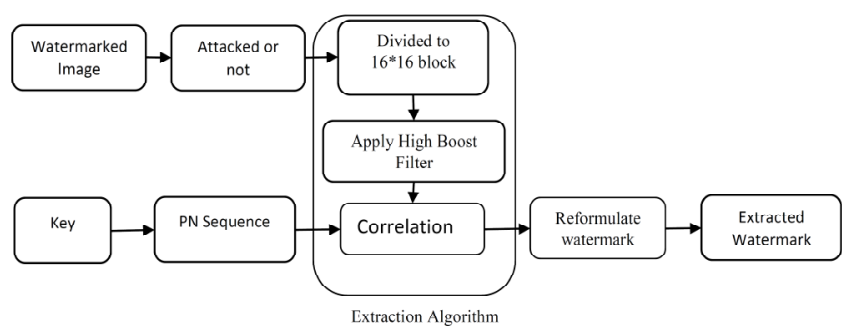

(b)

Figure 2. (a) The watermark extracting with applying High Boost before preforming extracting algorithm. (b) The watermark extracting process with applying High Boost in the middle of extracting algorithm. 


\section{Experimental Result}

To compare the efficiency of the proposed filter on correlation based methods, three standard grayscale images with different contents of size $512 \times 512$ are used in our experiments, as shown in ure $3(\mathrm{a})-(\mathrm{c})$. Pepper is used as a representation of image with low spatial frequency and Barbara as a representation of image with average spatial frequency and Baboon as a representation of image with high spatial frequency. In this experiment, a $32 \times 32$ binary image, as shown in Figure 3(d) is taken as the watermark of images. The effect of the High Boost filters is investigated by measuring imperceptible and robustness of watermarked image. For the imperceptible capability, a quantitative index, Peak Signal-to-Noise Ratio (PSNR), is employed to evaluate the difference between an original image $\mathrm{O}$ and a watermarked image. For the robust capability, Normalized Cross-Correlation (NC) measures the difference between an original watermark $\mathrm{W}$ and the corresponding extracted one. If a method has higher NC, it is more robust. The PSNR and the NC are, respectively, defined by equation 5 and 6; respectively,

$$
\begin{aligned}
& \operatorname{PSNR}(\mathrm{O}, \overline{\mathrm{O}})=10 \log _{10} \frac{255 \times 255}{\frac{\sum_{\mathrm{i}=0 \mathrm{j}=0}^{\mathrm{I}-1 \mathrm{~J}-1}\left(\left\|\mathrm{O}_{\mathrm{ij}}-\overline{\mathrm{O}}_{\mathrm{ij}}\right\|\right)^{2}}{\mathrm{I} \times \mathrm{J}}} \\
& N C=\frac{\sum_{i=1}^{M_{1}} \sum_{j=1}^{M_{2}} W(\mathrm{i}, \mathrm{j}) \cdot \mathrm{W}^{\prime}(\mathrm{i}, \mathrm{j})}{\sqrt{\sum_{i=1}^{M_{1}} \sum_{j=1}^{M_{2}}[W(\mathrm{i}, \mathrm{j})]^{2}} \sqrt{\sum_{i=1}^{M_{1}} \sum_{j=1}^{M_{2}}\left[W^{\prime}(\mathrm{i}, \mathrm{j})\right]^{2}}}
\end{aligned}
$$

Where $\|.\|_{1}$ and $|$.$| stand for the L1 norm, and the$ number of components of a vector, respectively.

The watermarked image $\mathrm{O}$ is obtained following the completion of the watermark embedding procedure. The watermark information is embedded with PSNR 30, 35, and $40 \mathrm{~dB}$ in the watermarked images. Then High Boost filter which is described in section 2 are performed on these watermarked images which may be attached by the method presented in section 3. NC between the original $\mathrm{W}$ and the extracted watermark $\mathrm{W}^{\prime}$ is calculated for different PSNR. To compare the effect of different places of preforming proposed filter, the algorithm is tested by several attacks, including JPEG compression, image scaling, adding Salt and Pepper noise, Gaussian filtering. The presented method is implemented using MATLAB.

\subsection{Visual Comparisons}

Table 1 shows visual comparisons between the effects of different position in convolving High Boost filter with watermarked image in the correlation based watermarked. These results have been gain form Pepper's image which watermarked with PSNR 40. As shown in the Table 1, although preforming the High Boost filter before correlation improve the trustworthiness of watermark retrieval of typical correlation based watermarking method, But, convolving the High Boost filter before running extraction slightly is better than it. These improvements in the case of scaling and blurring attacks are more significant than the others. From these experimental results, we could find that the convolving High Boost filter before running recovery algorithm is more applicable.

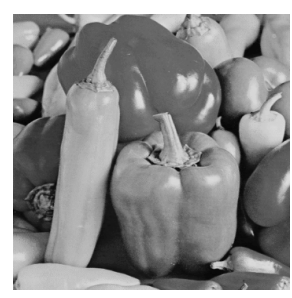

(a)

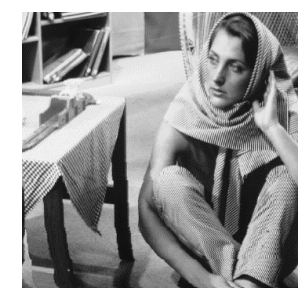

(b)

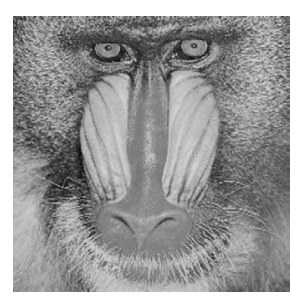

(c)

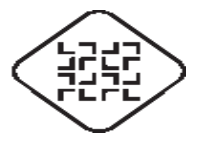

(d)

Figure 3. (a) The original Pepper image; (b) the original Barbara image; (c) the original Baboon image; (d) the original watermark. 
Table 1. Visual comparison between the extracted watermark from the peppers watermarked image with PSNR $=40$

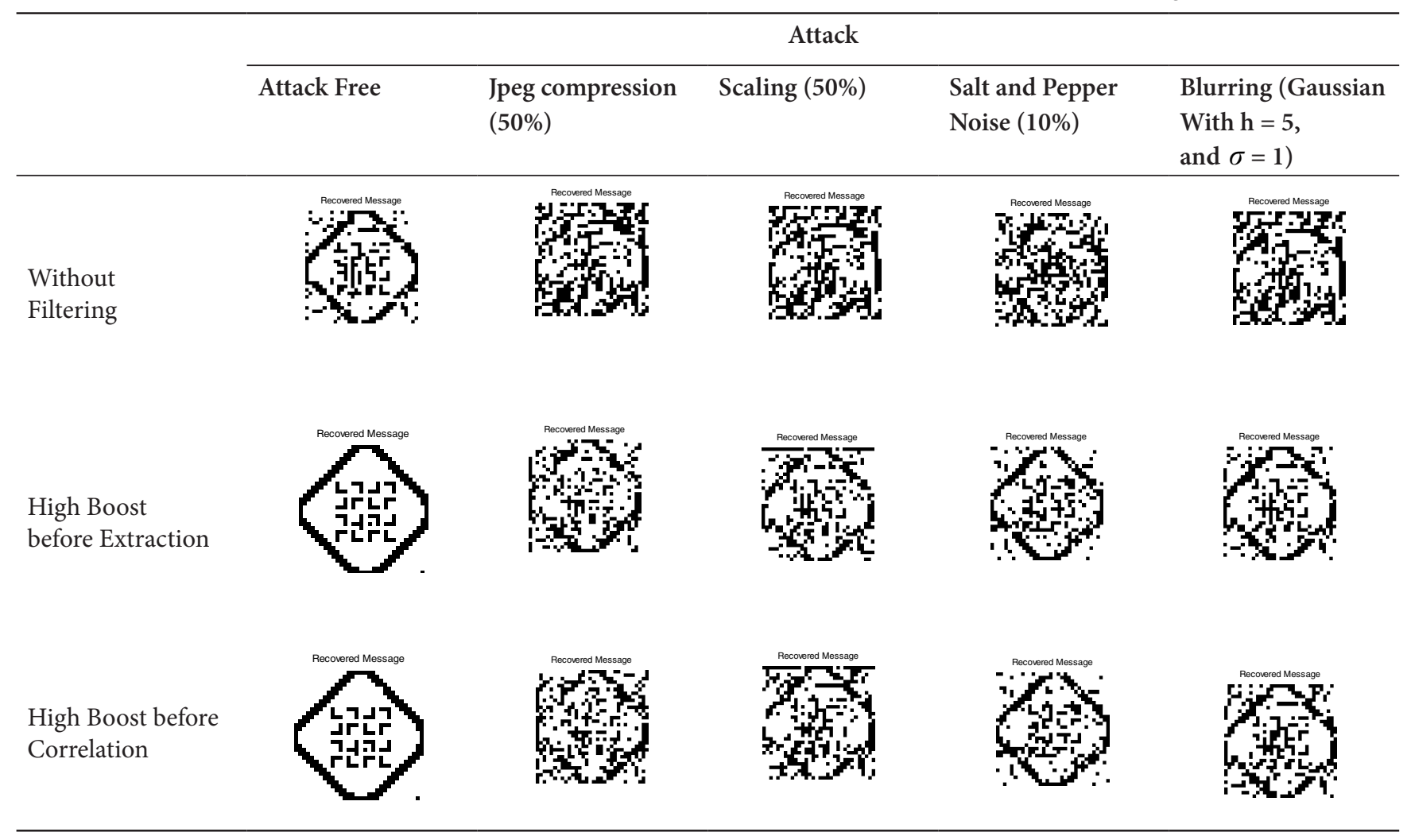

\subsection{Results for the Pepper Image}

In the second experiment, the results of preforming High Boost filter on Pepper image as a representation of image with low spatial frequency are presented. The goal of this section is to show the effect of different position of convolving this filter on a typical low spatial frequency image. As it is shown in the Table 2, results are meaningfully augmented by utilizing this filter in both of places for in compare with normal retrieval. Utilizing proposed filter before extraction algorithms is more effective to the attacked image with jpeg compression, scaling and blurring attacks. However, in the noise addition attacks, utilizing High Boost filter before calculating the correlation better increases the results. As it shown in this table, preforming High Boost filter before recovery algorithm shows better improvement in the values of NC. For example, in scaling attack on image with PSNR 40 as a high invisible watermarked image, NC preforms 0.027 much better than utilized this filter before calculating the correlation, and in the case of jpeg compression attack improvement is 0.02 in term of NC. Therefore, for low spatial frequency, using the High Boost before running extraction is more suitable than utilizing it before taking the correlation.

\subsection{Results for the Barbara Image}

In the third tests, the results of using High Boost filters on Barbara as a representation of image with average spatial frequency is shown. As shown in Table 3, results are significantly enhanced by utilizing proposed filter with the Barbara image in both position compared to normal recovery. Convolving High Boost filter before recovery algorithms is more successful to the attacked image with jpeg compression, scaling and blurring attacks. However, in the noise addition attack, using High Boost filter before taking the correlation better recovers the results. Applying High Boost filter before recovery algorithm shows better improvement in the values of NC. For example, in blurring attack on image with PSNR 40 as a high imperceptible watermarked image, NC preforms 0.02 much better than applying this filter before taking the correlation. Therefore, for average spatial frequency, convolving the High Boost before executing retrieval is more proper than using it before taking the correlation. 
Table 2. Comparison in term of NC between the recoverd watermark from the peppers watermarked image with diffrent methods

\begin{tabular}{|c|c|c|c|c|c|c|}
\hline \multirow[b]{2}{*}{ Pepper Image } & \multirow[b]{2}{*}{ PSNR } & \multicolumn{5}{|c|}{ Attack } \\
\hline & & Attack Free & $\begin{array}{l}\text { Jpeg } \\
\text { compression } \\
(50 \%)\end{array}$ & $\begin{array}{l}\text { Scaling } \\
(50 \%)\end{array}$ & $\begin{array}{l}\text { Salt and } \\
\text { Pepper } \\
\text { Noise }(10 \%)\end{array}$ & $\begin{array}{l}\text { Blurring } \\
\text { (Gaussian with } \\
\mathrm{h}=5 \text {, and } \sigma=1 \text { ) }\end{array}$ \\
\hline \multirow{3}{*}{ Without Filtering } & 30 & 0.9928 & 0.9274 & 0.8383 & 0.9154 & 0.8635 \\
\hline & 35 & 0.9743 & 0.8547 & 0.7856 & 0.8298 & 0.8105 \\
\hline & 40 & 0.9280 & 0.7464 & 0.7280 & 0.7336 & 0.7530 \\
\hline \multirow{3}{*}{$\begin{array}{l}\text { High Boost before } \\
\text { Extraction }\end{array}$} & 30 & 1 & 0.9967 & 0.9496 & 0.9961 & 0.9763 \\
\hline & 35 & 1 & 0.9608 & 0.9168 & 0.9875 & 0.9481 \\
\hline & 40 & 0.9974 & 0.8476 & 0.8596 & 0.9070 & 0.8904 \\
\hline \multirow{3}{*}{$\begin{array}{l}\text { High Boost before } \\
\text { Correlation }\end{array}$} & 30 & 1 & 0.9948 & 0.9330 & 0.9980 & 0.9749 \\
\hline & 35 & 1 & 0.9512 & 0.8905 & 0.9862 & 0.9439 \\
\hline & 40 & 0.9974 & 0.8257 & 0.8318 & 0.9185 & 0.8744 \\
\hline
\end{tabular}

Table 3. comparison in term of NC between the extracted watermark from the Barbara watermarked image with diffrent methods

\begin{tabular}{lcccccc}
\hline & & \multicolumn{5}{c}{ Attack } \\
\cline { 3 - 7 } Barbara Image & PSNR & Attack Free & $\begin{array}{l}\text { Jpeg } \\
\text { compression } \\
(50 \%)\end{array}$ & Scaling (50\%) & $\begin{array}{l}\text { Salt and } \\
\text { Pepper } \\
\text { Noise (10\%) }\end{array}$ & $\begin{array}{l}\text { Blurring } \\
\text { (Gaussian with } \\
\mathbf{h}=5 \text {, and } \sigma=1)\end{array}$ \\
\hline Without Filtering & 30 & 0.9875 & 0.9072 & 0.8033 & 0.9170 & 0.8332 \\
& 35 & 0.9400 & 0.8044 & 0.7549 & 0.8077 & 0.7762 \\
High Boost before & 30 & 0.8608 & 0.7054 & 0.7214 & 0.7198 & 0.7336 \\
Extraction & 30 & 0.9967 & 0.9762 & 0.9135 & 0.9763 & 0.9502 \\
& 35 & 0.9842 & 0.8969 & 0.8521 & 0.9384 & 0.8882 \\
High Boost before & 30 & 0.9446 & 0.7835 & 0.7849 & 0.8497 & 0.8187 \\
Correlation & 35 & 0.9836 & 0.8865 & 0.8307 & 0.9419 & 0.9481 \\
& 40 & 0.9500 & 0.7682 & 0.7611 & 0.8458 & 0.8751 \\
\hline
\end{tabular}

\subsection{Results for the Baboon Image}

In the fourth experiment, the results of applying High Boost filter on Baboon image as a representation with high spatial frequency are shown. Results are considerably augmented by convolving of the High Boost filter with the watermarked image in both locations in compare with standard retrieval (Table 4). Convolving proposed filter before retrieval algorithms is more effective in case of jpeg compression and blurring attacks and to somewhat in the scaling attack. However, in the noise addition attack, using proposed filter before taking the correlation better increases the results slightly. Therefore, for high spatial frequency, convolving the High Boost before running recovery and before taking the correlation, have the same performance.

\section{Conclusions}

In this paper, comparison is made between the effects of different locations of using High Boost filter on the watermarked image in increasing the power of watermark recovery algorithms. The High Boost filter is convolved 
Table 4. comparison in term of NC between the extracted watermark from the Baboon watermarked image with diffrent methods

\begin{tabular}{|c|c|c|c|c|c|c|}
\hline \multirow{2}{*}{ Baboon Image } & \multirow{2}{*}{ PSNR } & \multicolumn{5}{|c|}{ Attack } \\
\hline & & Attack Free & $\begin{array}{l}\text { Jpeg compression } \\
(50 \%)\end{array}$ & $\begin{array}{l}\text { Scaling } \\
(50 \%)\end{array}$ & $\begin{array}{l}\text { Salt and Pepper } \\
\text { Noise }(10 \%)\end{array}$ & $\begin{array}{l}\text { Blurring (Gaussian } \\
\text { With } \mathrm{h}=5 \text {, and } \sigma=1 \text { ) }\end{array}$ \\
\hline \multirow{3}{*}{ Without Filtering } & 30 & 0.9993 & 0.9696 & 0.8291 & 0.9403 & 0.8677 \\
\hline & 35 & 0.9743 & 0.8883 & 0.7534 & 0.8385 & 0.7843 \\
\hline & 40 & 0.9330 & 0.8206 & 0.7176 & 0.7783 & 0.7425 \\
\hline \multirow{3}{*}{$\begin{array}{l}\text { High Boost before } \\
\text { Extraction }\end{array}$} & 30 & 1 & 0.9875 & 0.9148 & 0.9928 & 0.9716 \\
\hline & 35 & 0.9888 & 0.9312 & 0.8340 & 0.9500 & 0.9011 \\
\hline & 40 & 0.9674 & 0.8550 & 0.7806 & 0.8976 & 0.8525 \\
\hline \multirow{3}{*}{$\begin{array}{l}\text { High Boost before } \\
\text { Correlation }\end{array}$} & 30 & 1 & 0.9855 & 0.9083 & 0.9941 & 0.9621 \\
\hline & 35 & 0.9915 & 0.9265 & 0.8349 & 0.9540 & 0.8966 \\
\hline & 40 & 0.9688 & 0.8547 & 0.7780 & 0.9049 & 0.8448 \\
\hline
\end{tabular}

with watermarked image in two locations; before running retrieval algorithm, and in the middle of retrieval and before taking the correlation. Several tests are done to investigate which of these locations is more suitable for enhancing spatial domain watermarking method. Efficiency of the methods is tested by comparing its results with each other in the term of NC. The watermark is extracted after common image processing attacks with higher NC value by using High Boost filter before retrieval algorithm. Especially, performance is increased better in case of blurring filter, jpeg compression and scaling. The images with high frequency results are almost identical. However, results of preforming High Boost filter before taking the correlation is better than applying filter before executing recovery algorithm, in the case of salt and pepper noise. Therefore, we suggest to use High Boost filter before running watermark retrieval algorithms in the spatial domain watermarking algorithm.

\section{References}

1. Amirgholipour SK, Naghsh-Nilchi AR. Robust digital image watermarking based on joint DWT-DCT. Journal of Digital Content Technology and its Applications. 2009; 3(2):42-54.

2. Kasmani SA, Naghsh-Nilchi A. A new robust digital image watermarking technique based on joint DWT-DCT transformation. Third International Conference on Convergence and Hybrid Information Technology. ICCIT'08. 2008 Nov. IEEE. p. 539-44

3. Kasmani SA, Mahfouzi M, Asfia M. A new pre-processing approach to improve DCT-Based Watermarkings Extraction. International Association of Computer Science and Information Technology-Spring Conference. IACSITSC'09. 2009 Apr; IEEE. p. 131-35.

4. Potdar VM, Han S, Chang E. A survey of digital image watermarking techniques. $3^{\text {rd }}$ IEEE International Conference on Industrial Informatics. INDIN'05. 2005 Aug. IEEE. p. $709-16$.

5. Kasmani SA, Sharifi AM. A pre-filtering method to improve watermark detection rate in DCT based watermarking. Int Arab J Inform Tech. 2014 Mar; 11(2):178-185.

6. Depovere G, Kalker T, Linnartz JP. Improved watermark detection reliability using filtering before correlation. International Conference on Image Processing. ICIP 98. 1998 Oct. IEEE. p. 430-34.

7. Chu WC. DCT-based image watermarking using subsampling. IEEE Trans Multimed. 2003; 5(1):34-8.

8. Lin SD, Chen CF. A robust DCT-based watermarking for copyright protection. IEEE Trans Consum Electron. 2000; 46(3):415-21.

9. ûHsieh MS, Tseng DC, Huang YH. Hiding digital watermarks using multiresolution wavelet transform. IEEE Trans Ind Electron. 2001; 48(5):875-82.

10. Reddy AA, Chatterji BN. A new wavelet based logowatermarking scheme. Pattern Recogn Lett. 2005; 26(7):1019-27. 
11. Watson AB. Perceptual optimization of DCT color quantization matrices. IEEE International Conference on Image Processing. ICIP-94; 1994 Nov. IEEE. p. 100-04.

12. Chou CH, Li YC. A perceptually tuned subband image coder based on the measure of just-noticeable-distortion profile. IEEE Trans Circ Syst Video Tech. 1995; 5(6):467-76.

13. Podilchuk CI, Zeng W. Image-adaptive watermarking using visual models. IEEE J Sel Area Comm. 1998; 16(4):525-39.

14. Barni M, Bartolini F, Piva A. Improved wavelet-based watermarking through pixel-wise masking. IEEE Trans Image Process. 2001; 10(5):783-91.

15. Kutter M, Winkler S. A vision-based masking model for spread-spectrum image watermarking. IEEE Trans Image Process. 2002; 11(1):16-25.

16. Levický D, Foriš P. Human visual system models in digital image watermarking. Radioengineering. 2004; 13(4):38-43.
17. Fotopoulos V, Skodras AN. Improved watermark detection based on similarity diagrams. Signal Process Image Comm. 2002; 17(4):337-45.

18. Braudaway GW, Mintzer FC. Application of blurring filters toimprovedetectionofinvisibleimagewatermarks.Proceedings of SPIE; 2003 Jun. p. 269-77.

19. Malik H, Khokhar A, Ansari R. Improved watermark detection for spread-spectrum based watermarking using independentcomponentanalysis.Proceedingsofthe 5thACM workshoponDigitalrightsmanagement.2005Nov.p.102-11.

20. Pan Z, Li L, Zhang M, Zhang D. Watermark extraction by magnifying noise and applying global minimum decoder. Third International Conference on Image and Graphics. 2004 Dec. p. 349-52.

21. Gonzalez RC, Woods RE. Digital Image Processing: Introduction; 2002. 